\title{
Mice developing mammary tumors evolve $T$ cell sequences shared with human breast cancer patients
}

\author{
Miri Gordin ${ }^{1 \dagger}$, Hagit Philip ${ }^{1 \dagger}$, Alona Zilberberg${ }^{1}$, Moriah Gidoni ${ }^{2}$, Raanan Margalit ${ }^{3}$, Christopher Clouser ${ }^{4}$, \\ Kristofor Adams ${ }^{4}$, Francois Vigneault ${ }^{4}$, Irun R. Cohen ${ }^{5}$, Gur Yaari ${ }^{2 *}$, Sol Efroni ${ }^{1 *}$
}

1 The Mina \& Everard Goodman Faculty of Life Sciences, Bar Ilan University, Ramat-Gan, Israel. ${ }^{2}$ Faculty of Engineering, Bar Ilan University, Ramat Gan, Israel. ${ }^{3}$ Science in Action Ltd., Ness Ziona, Israel. ${ }^{4}$ Juno Therapeutics, Seattle, WA, USA. ${ }^{5}$ Department of Immunology, The Weizmann Institute of Science, Rehovot, Israel. ${ }^{*}$ Corresponding authors. Email: sol.efroni@biu.ac.il, gur.yaari@biu.ac.il ${ }^{\dagger}$ These authors contributed equally to this work.

\begin{abstract}
Cancer immunotherapy by checkpoint blockade proves that an effective immune response to a tumor can be induced clinically. However, little is known about the evolution of tumorassociated T-cell receptor (TCR) repertoires without intervention. Here we studied TCR repertoire evolution in mice spontaneously developing mammary tumors; we sequenced peripheral blood alpha and beta TCRs of $\mathrm{CD} 4^{+} \mathrm{CD} 62 \mathrm{~L}^{+} \mathrm{CD} 44^{-} \mathrm{T}$ cells monthly for 8 months in $10 \mathrm{FVB} / \mathrm{NJ}$ mice transgenic at the Erbb2 locus, all developing tumors; $5 \mathrm{FVB} / \mathrm{NJ}$ mice without the transgene were age-matched controls. Sequences were either private (restricted to one mouse) or public (shared among mice); public sequences were either exclusive to the tumor group or inclusive among different groups. We now report that 1), public AA sequences were each encoded by many different nucleotide sequences (NT) recombinations (convergent recombination; CR); 2) mice developing tumors evolved tumor-exclusive public sequences, derived initially from private or from inclusive public sequences; and 3) tumor-exclusive public sequences in mice were also present among published public TCR sequences from human breast cancer patients. These cross-species tumor-exclusive TCR sequences manifested high CR; but the AA sequences shared by mice and humans did not share NT sequences. Thus, tumor-exclusive TCR AA sequences across species are selected from different NT recombination events. The roles of tumor-exclusive TCR repertoires in advancing or inhibiting tumor development and the effects of tumor immunotherapy on these $\mathrm{T}$ cells remain to be seen.
\end{abstract}

\section{Introduction}

$\mathrm{T}$ cells control multiple immune mechanisms that are involved in tumor progression (Dunn, Bruce, Ikeda, Old, \& Schreiber, 2002; Hanahan \& Weinberg, 2011). Attempts to influence the interaction between T cells and cancer cells often rely on specific peptide presentation on the surface of tumor cells or of antigen presenting cells (Butterfield, 2015; Kawakami et al., 1994; Simpson, Caballero, Jungbluth, Chen, \& Old, 2005; Slingluff, 2011), the presentation of a relevant receptor on the surface of an interacting T cell (Brentjens et al., 2013; Ye et al., 2018), or on the manipulation of co-stimulation of the interaction (Drake, Lipson, \& Brahmer, 2014; Hoos et al., 2010; Pardoll, 2012; Sonpavde, 2017). Each T cell clone is characterized by a heterodimeric T-cell Receptor (TCR), and its interaction with a (tumor) cell is further characterized by a protein degradation product presented by the major histocompatibility complex (MHC) on the cell's surface. For most T-cells, the heterodimeric TCR is composed of $\alpha$ (TRA) and $\beta$ (TRB) chains (less than $10 \%$ express the $\gamma$ and $\delta$ form of TCR(Kalyan \& Kabelitz, 2013) ). The sequences of TRB chains are ultimately determined by somatic $\mathrm{V}(\mathrm{D}) \mathrm{J}$ recombination of the Variable (V), Diversity (D) and Joining (J) paralogs, and the recombination of $\mathrm{VJ}$ for the TRA chain. This recombination includes addition, deletion, and replacement of nucleotides, which leads to extreme possible variability of the complementarity-determining region 3 (CDR3) loop, that interacts with the MHC presented peptides (Pellicci et al., 2014). The collection of available receptors on the surface of $\mathrm{T}$ cells has been termed the T-cell repertoire (Fukui et al., 1998). T cells make up to $60 \%$ of PBMCs, so that even small volumes of drought peripheral blood, contain a large number of $\mathrm{T}$ 
bioRxiv preprint doi: https://doi.org/10.1101/371260; this version posted August 6, 2018. The copyright holder for this preprint (which was not certified by peer review) is the author/funder. All rights reserved. No reuse allowed without permission.

cells, such that a milliliter of peripheral blood contains 0.5-1.5 million T cells (Pagana \& Pagana, 2007). T cells are early responders to neoplasm, and we set out to find if changes to the $\mathrm{T}$ cell repertoire, previously demonstrated in tumor (Galon et al., 2006; Ino et al., 2013; Zhang et al., 2003), are also quantifiable in the periphery. The use of peripheral blood, instead of other tissue types, allows the unique capability of sampling over time points along tumor progression. Such information flow, out of the tumor, and into repertoire data, is taking place through multiple channels. Blood flow in and out of the tumor provides direct tethering between different $\mathrm{T}$ cell compartments, and other channels of control over the CDR3 repertoire include interactions between multiple arms of the immune system that interact with the tumor microenvironment (Gajewski, Schreiber, \& Fu, 2013).

The co-evolution of the $\mathrm{T}$ cell repertoire and a neoplastic tumor over a significant period ( 9 months) of mice life is accompanied by the evolution of the TCR repertoire during mice maturation and mice ageing. It has already been demonstrated that the $\mathrm{T}$ cell repertoire changes significantly with age (Britanova et al., 2014; Yoshida et al., 2017). To control for this factor, we used mice with the same genetic background as the transgenic mice, but are non-transgenic and do not develop tumors.

\section{Results}

We followed the development of spontaneous tumors in a transgenic mouse expressing the inactivated rat neu (Erbb2) oncogene. This mouse model serves as a model of HER2+ human breast cancer (Guy et al., 1992). As a control, we use FVB/NJ strain mice with the same genetic background, but lacking the transgene. We sequenced TCR repertoires in the peripheral bloods of each of the 15 mice drawn at 8 monthly time points (Figure 1). We focused on the largest recoverable subset of $\mathrm{T}$ cells, those bearing markers $\mathrm{CD} 4{ }^{+} \mathrm{CD} 62 \mathrm{~L}^{+} \mathrm{CD} 44^{-}$. The counts of TCR $\alpha$ and TCR $\beta$ sequences are presented in Supplementary Table 1. We categorized the following groups: Young (mice 2-5 months old); Old (mice 6 months or older); Control; and Transgenic (Tg): pretumor; early-tumor (one month before a palpable tumor); and tumor. Supplementary Table 1 tags samples by these categories.

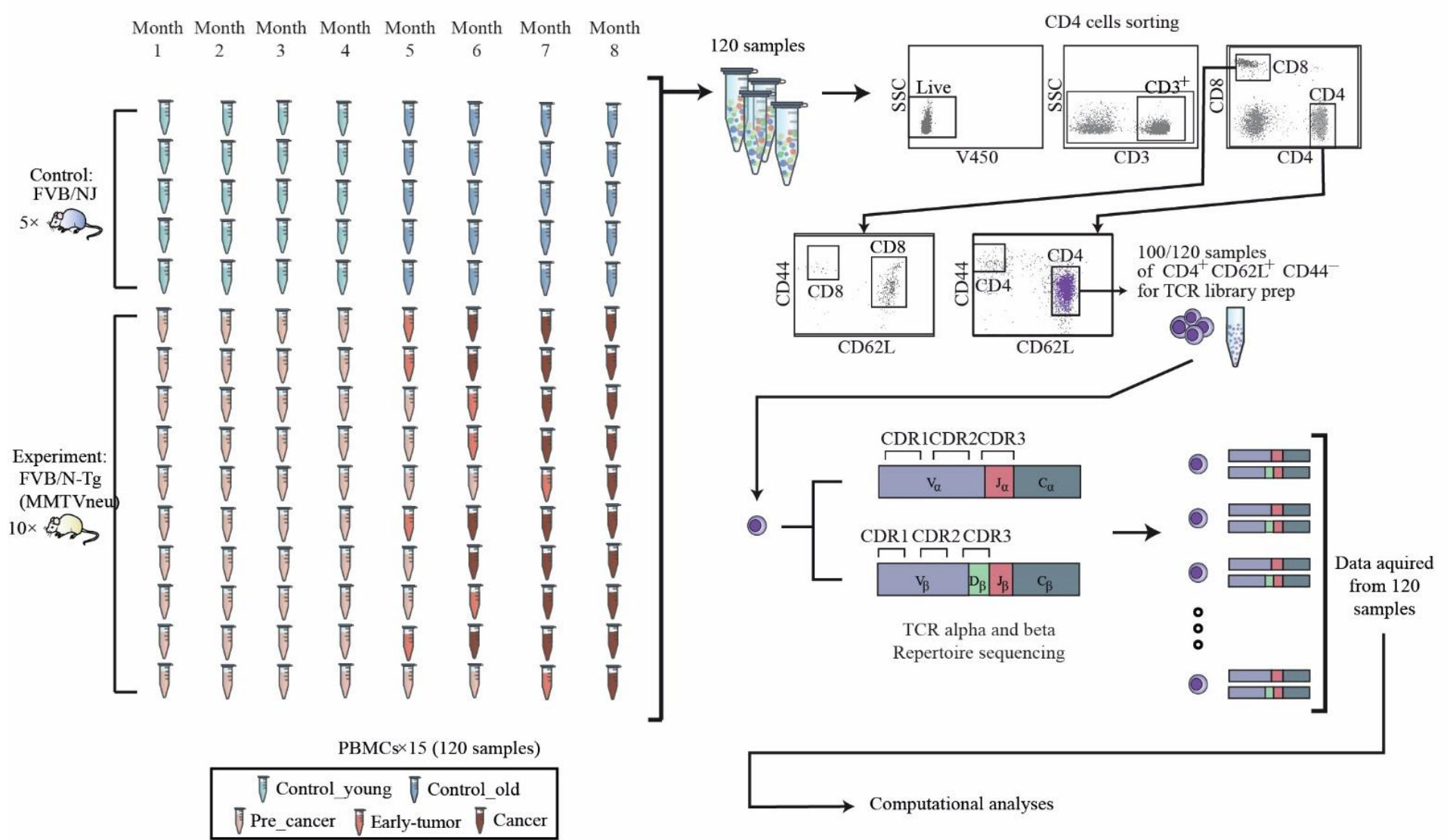

Fig. 1. Experimental procedure.

120 blood samples were drawn from the retro-orbital sinus of $10 \mathrm{FVB} / \mathrm{N}-\mathrm{Tg}$ (MMTVneu), a mouse model of HER2 human breast cancer mice, and from 5 FVB/NJ control mice. Over these 8 time-points, none of the control mice (blue) developed any tumors. Progress of tumor in the ten transgenic mice is demonstrated using the red colored samples in the figure. The last time point before tumors are shown was defined as pre-cancer and marked light red. From each time point, the peripheral blood mononuclear cells were isolated and stained for flow cytometry. Cells were analyzed and gated for sorting using a FACS ARIA III sorter, and CD4+CD62L+CD44-nä̈ve population was separated for RNA extraction and T cell receptor library preparation (see Methods). 
We found that there were no significant differences in repertoire diversity or CDR3 length distributions; but there were differences in copy number and percent of unique sequences among the groups (Supplementary Fig. 1). Private clonotypes refer to CDR3 AA sequences appearing in only one mouse; public clonotypes appear in at least two different mice. Public clonotypes are termed Inclusive if they appear in more than one phenotype and Exclusive if they are shared only by mice of the same phenotype group. We found that Exclusive Public clonotypes are more prevalent in the Tg group (19-23\%) compared to the Control group (3-5\%; Fig. 2a and Supplementary Fig. 2a). In other words, tumor-exclusive public sequences mark the $\mathrm{Tg}$ mice. Interestingly, public clonotypes occupy between $40-60 \%$ of the sequences in all the phenotypes.

Convergent recombination (CR) refers to identical TCR $\alpha$ or TCR $\beta$ amino-acid (AA) sequences originating from different nucleotide (NT) sequences. We found a higher frequency of CR in the $\alpha$ chain $(56 \%$ 69\%; Supplementary Fig. 3a) compared to the $\beta$ chain (43\%-52\%; Supplementary Fig. 3b). Figures $2 \mathrm{a}$ and $2 \mathrm{~b}$ show that $\mathrm{CR}$ in the Inclusive Public groups is double that of the Private groups; Figures $2 \mathrm{c}$ and $2 \mathrm{~d}$ show that the Exclusive-Public clonotypes originated from a smaller portion of the private clones in the Control group compared to the $\mathrm{Tg}$ group. In the private groups, the nonCR clonotypes dominated the repertoire (Supplementary Fig.3c, 3d). In addition, we identified a significant contribution of CR to the public TCR repertoire - 97\%$99 \%$ of the CR clonotypes of the $\alpha$ chain are public (Fig. 2e) compared to $13 \%-28 \%$ of public clonotypes in nonCR clonotypes (Supplementary Fig.3c). The same phenomenon is observed in $\beta$ chain $-96 \%-99 \%$ of the CR clonotypes are also public clonotypes (Fig. 2f), in comparison to $6 \%-26 \%$ of public clonotypes in the nonCR clonotypes (Supplementary Fig. 3d).

As CR levels increased, the sharing level for all groups increased (Fig. 2g, 2h). Furthermore, the connection between CR and sharing is group specific. In the $\mathrm{Tg}$ group, sharing increases faster when $\mathrm{CR}$ increases, compared with the Control group (Fig. $2 \mathrm{~g}$ ). This may indicate that selection (associated with CR levels) of specific clonotypes, is dominant in the transgenic mice, perhaps due to the sharing of tumor antigens among mice. Separating the transgenic group into pre-tumor and tumor samples, we see that sharing is greater in the pre-tumor group than in the tumor group (Fig. 2h); thus, high CR and highly shared sequences develop early, before we detect a palpable tumor.

To learn whether our observations in the mouse model might be relevant to human breast cancer, we compared the mouse TCR data to three, previously published human TCR datasets, each of which focused on a different aspect of breast cancer (Figure 3a) (Beausang et al., 2017; Page et al., 2016; Wang et al., 2017): Beausang et al (Beausang et al., 2017) studied $T$ cell receptors in early-stage breast cancer and sampled the tumor tissue, the surrounding normal tissue and peripheral blood. Wang et al (Wang et al., 2017) sequenced the TCR repertoires of breast cancer tumors, lymph nodes and adjacent tissue. Page et al (Page et al., 2016) studied breast cancer after immunotherapy. We asked two questions: do mice and humans with breast cancer share cross-species TCR repertoires, and do these cross-species TCRs manifest common CR events.

As we shall elaborate in greater detail below, mice and humans with breast cancer do share TCR CDR3 AA sequences. Figure $3 \mathrm{~b}$ compares mousespecific NT and AA sequences (inner circle) with crossspecies mouse sequences shared with human patients (outer circle); the groups are divided into Private, Public Inclusive and Public Exclusive. Regarding NT sequences (the left two wheels), it can be seen that the $\mathrm{Tg}$ mice express more Public Exclusive and less Public Inclusive sequences than did the Control mice. AA sequences, in contrast, manifest a much greater proportion of Public Inclusive sequences than Public Exclusive sequences. Nevertheless, Tg mice do express relatively more Public Exclusive sequences. Thus, NT and AA sequences appear to be unconnected generally, and mice developing tumors develop relatively more Public Exclusive sequences of both NT and AA types.

Figure $3 \mathrm{~d}$ shows that cross species clonotypes shared by mice and humans, both Control and Tg, manifest greater frequencies of $\mathrm{CR}$ than do mouse-specific sequences.

Figure 3e, compares the Control and $\mathrm{Tg}$ mouse groups to each of the three human studies regarding Tumor, nontumor and normal tissues. It can be seen that in each case the $\mathrm{Tg}$ mouse sequences manifested more sharing with the human repertoires than did the Control sequences. Tumor development appears to amplify the proportion of mouse TCR clonotypes shared with human breast cancer patients, even in non-tumor tissue. Note that the human non-tumor samples were obtained from cancer patients; there was no human Control group equivalent to the mouse Control group.

To obtain an additional metric to match samples across species, we used a ranking system to compare mouse sequences with sequences obtained from earlystage breast cancer patients reported by Beausang et al. First, we filtered for the set of sequences that appear in all mouse time-points and in the human samples. This resulted in 258 CDR3 cross-species sequences. At each time-point, we ranked the sequences according to their copy number -the highest copy number was ranked 1 , he second highest ranked 2 and so on. We then asked if the ranking in the human samples was similar to the ranking in the mouse sample, at each time point. To quantify these differences, we produced the bar-chart of Figure $3 \mathrm{~g}$. Every time-point in the bar-chart contains a stack of 258 bars. The area each bar occupies in the stack-bar is inversely proportional to an area defined by the ranking of the sequence at the specific time-point, multiplied by the ranking of the sequence in the human samples, such 
A

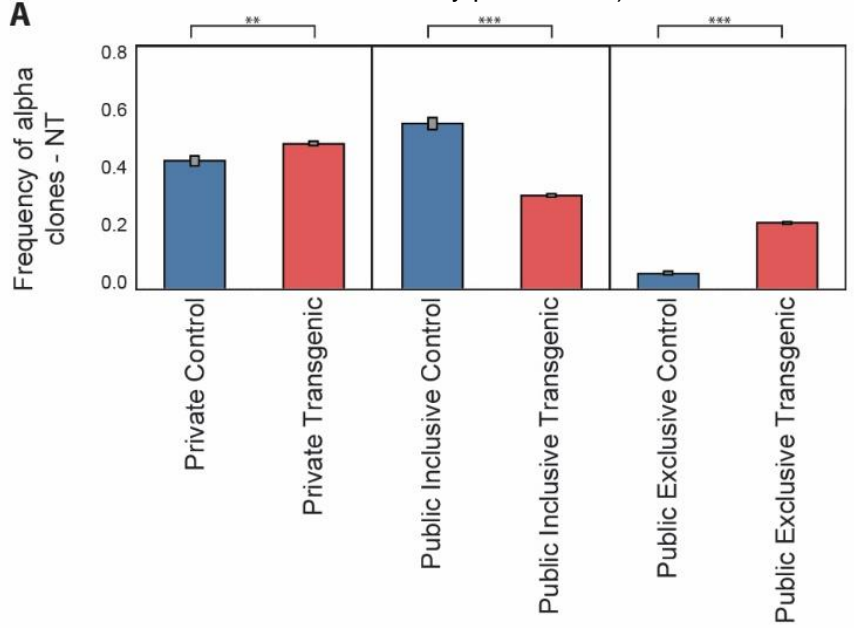

C

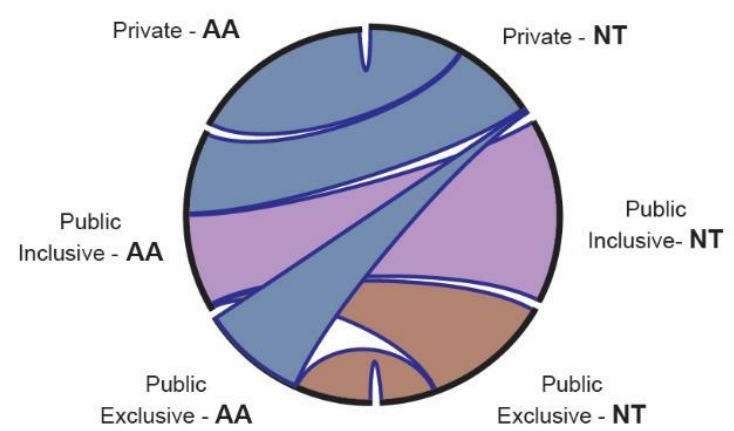

E

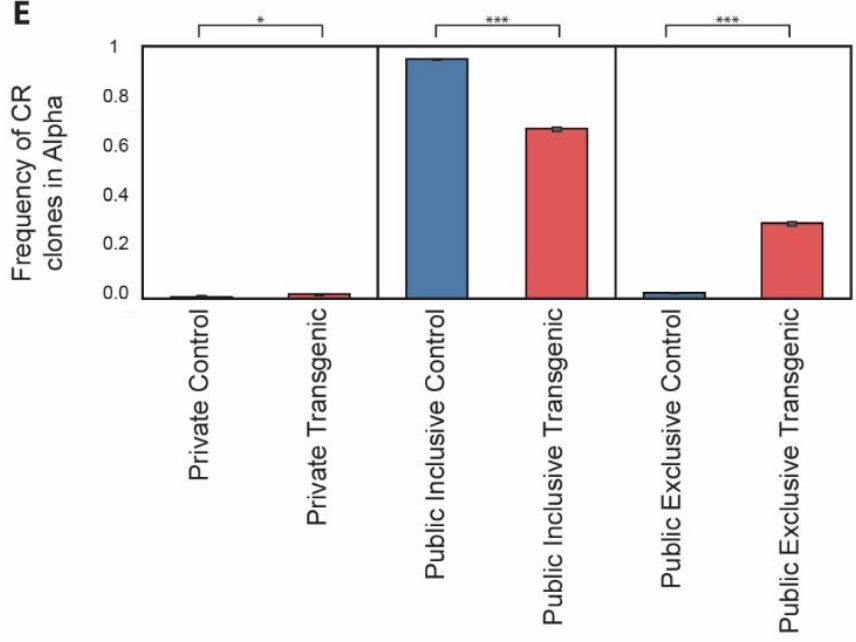

G

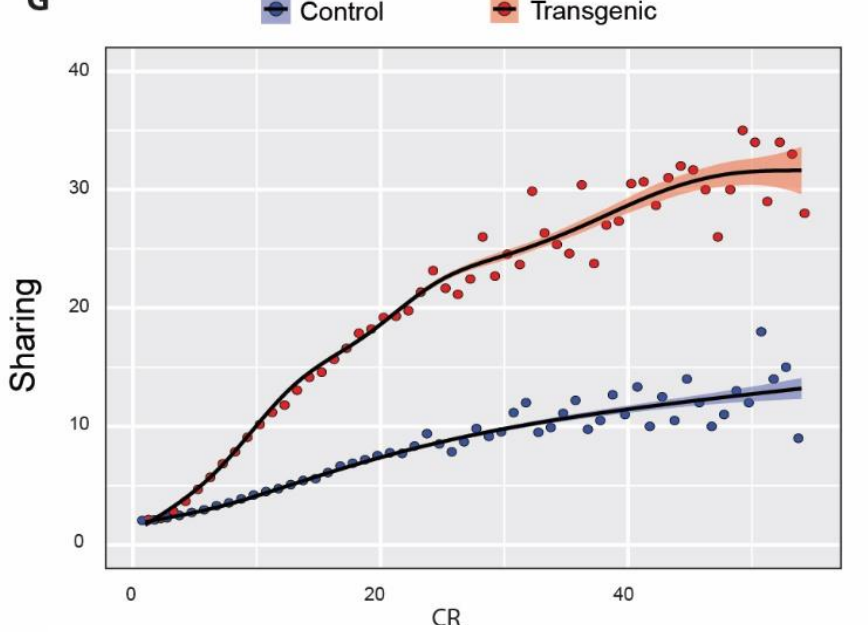

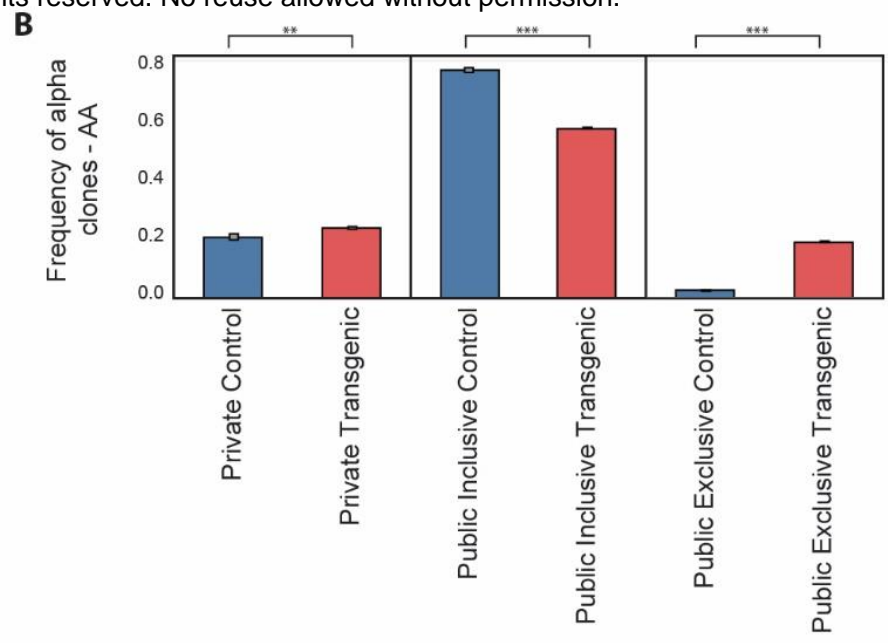

D

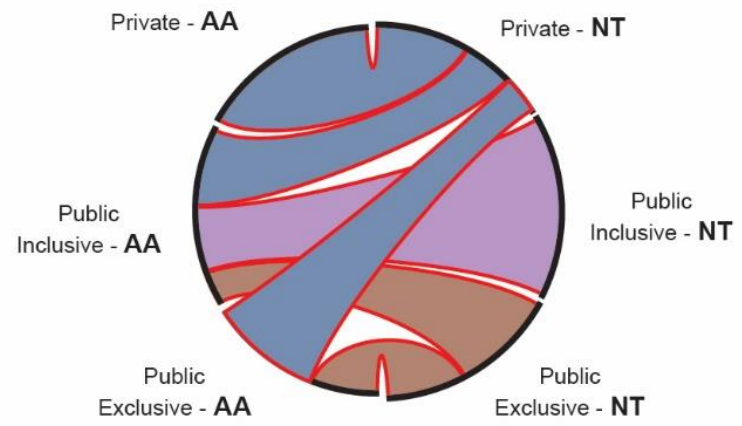

$\mathbf{F}$

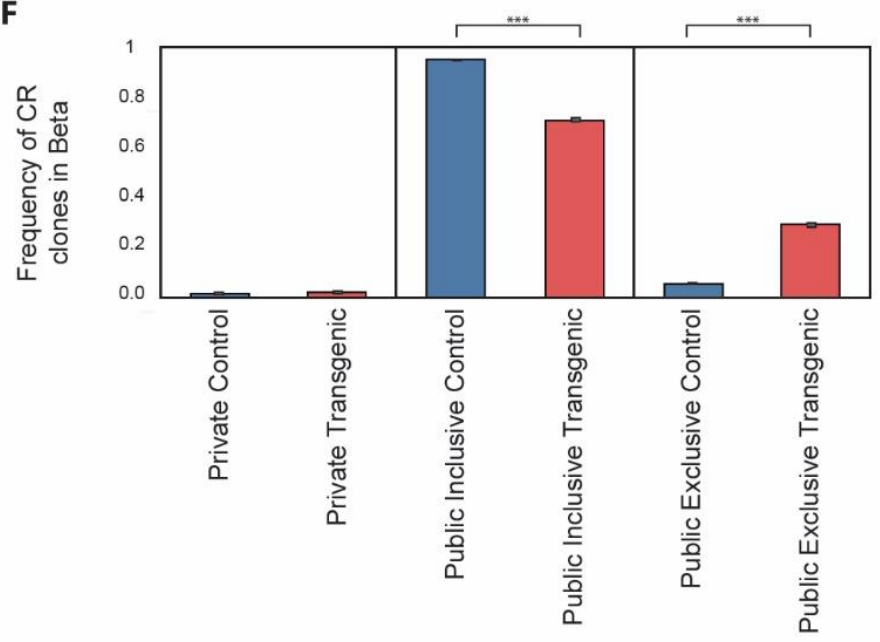

H Transgenic $\quad$ Pre-cancer $\quad$ Cancer

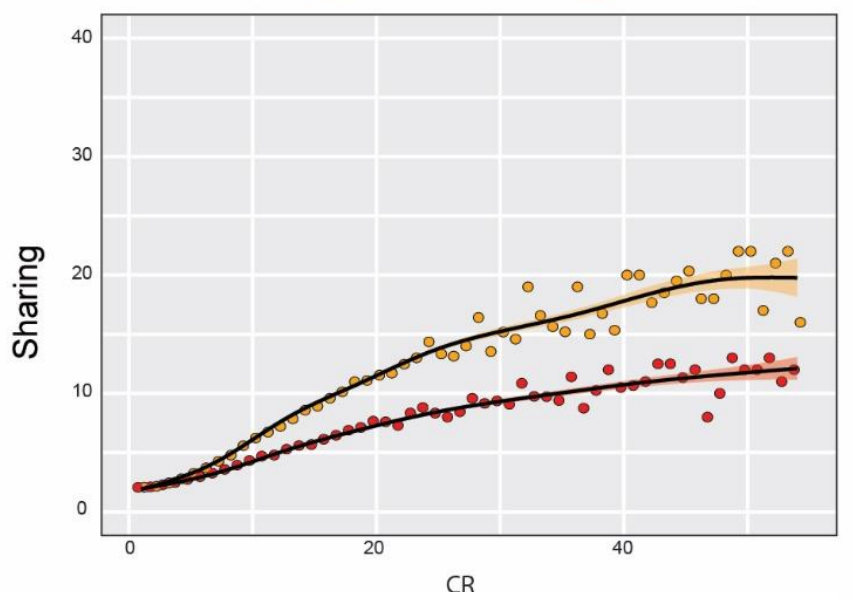

Fig. 2. The public repertoire and convergent recombination. Public repertoires and their subtypes are shown in Tg mice and in Control mice using nucleotide sequences $(\boldsymbol{A})$ and using amino-acid sequences $(\boldsymbol{B})$. The two panels show the repertoire in the alpha chain, but a similar effect is seen in the beta chain (Supplementary Fig. 2). (C, D) Convergent recombination in control and transgenic mice. The right-hand side of each circle indicates nucleotide (NT) sequences, while the left-hand side indicates amino-acid (AA) sequences. The edges between NT and AA represent the effect we see in convergent recombination - in which different NT sequences encode to the same AA sequence and change the Public/Private balance. $(\boldsymbol{E}, \boldsymbol{F})$ Frequencies of CR clones in the public repertoire in alpha and beta chains. $(\boldsymbol{G}, \boldsymbol{H})$ Correlation between sharing level and $C R$ level in Control versus $T g$ groups $(G)$ and in No-Cancer group vs. Cancer group (H). 
bioRxiv preprint doi: https://doi.org/10.1101/371260; this version posted August 6,2018 . The copyright holder for this preprint (which was not certified by peer review) is the author/funder. All rights reserved. No reuse allowed without permission.

A
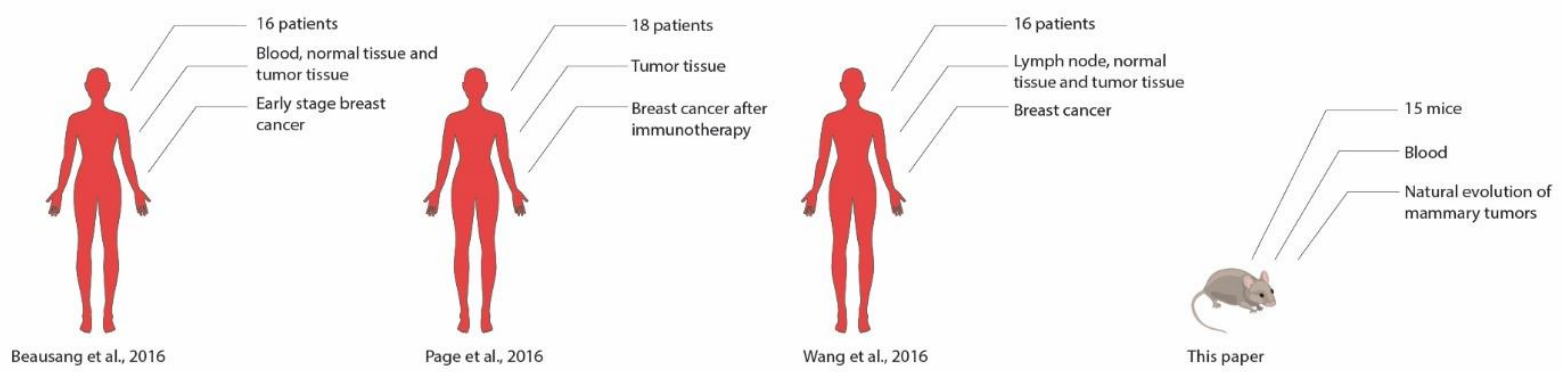

B

Control-NT

Transgenic - NT

C

Control - AA

Transgenic - AA
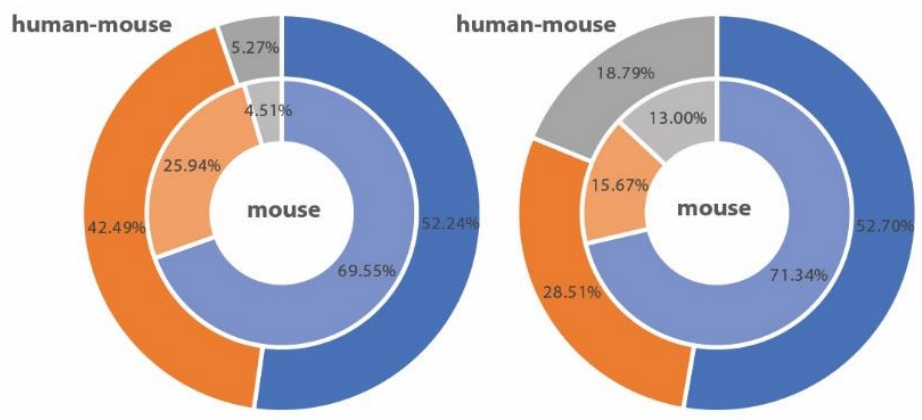

human-mouse

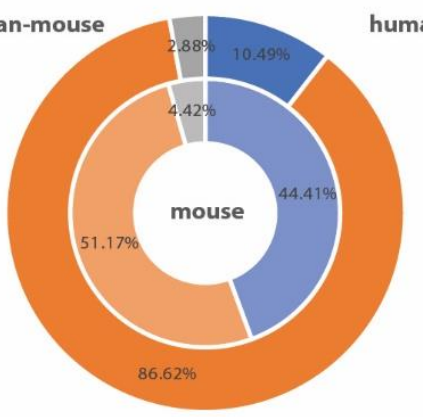

human-mouse

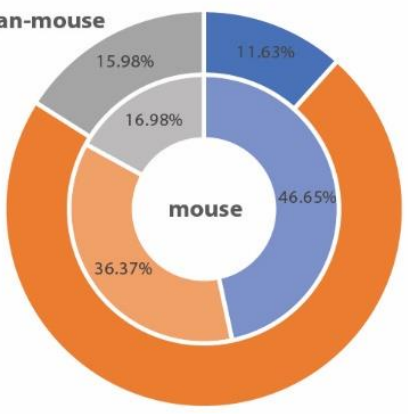

- Private $\quad$ Public Inclusive $\quad$ Public Exclusive

D

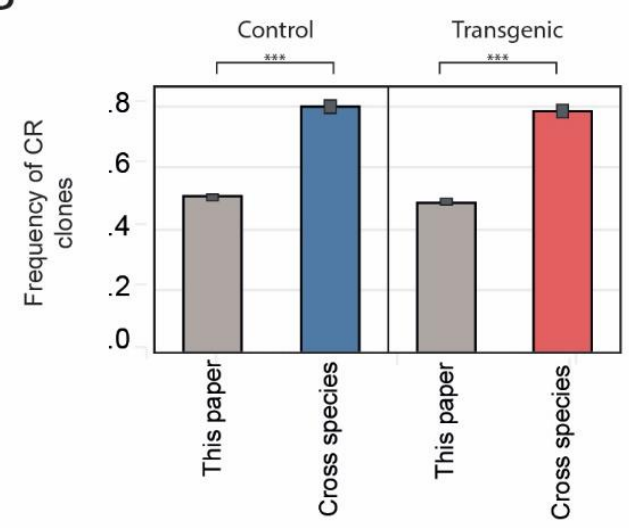

F

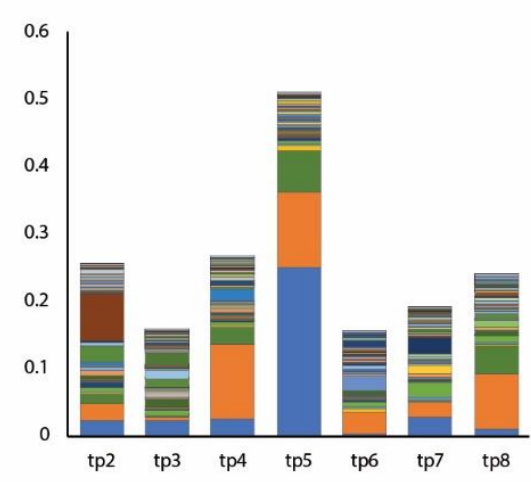

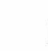

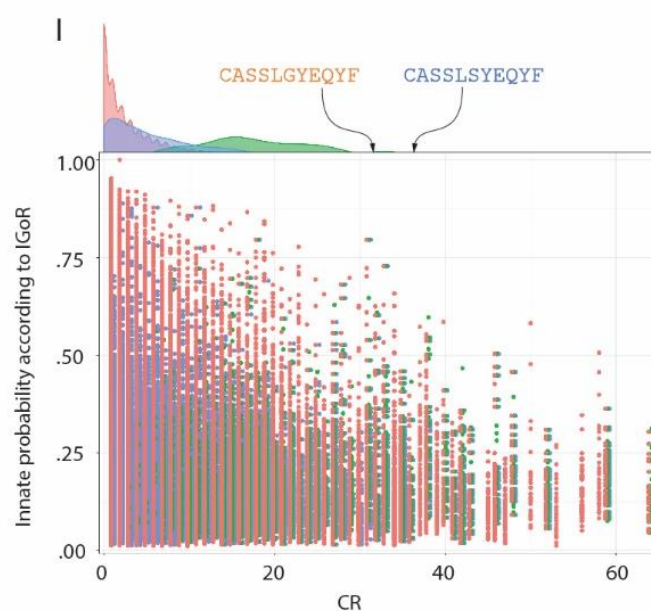

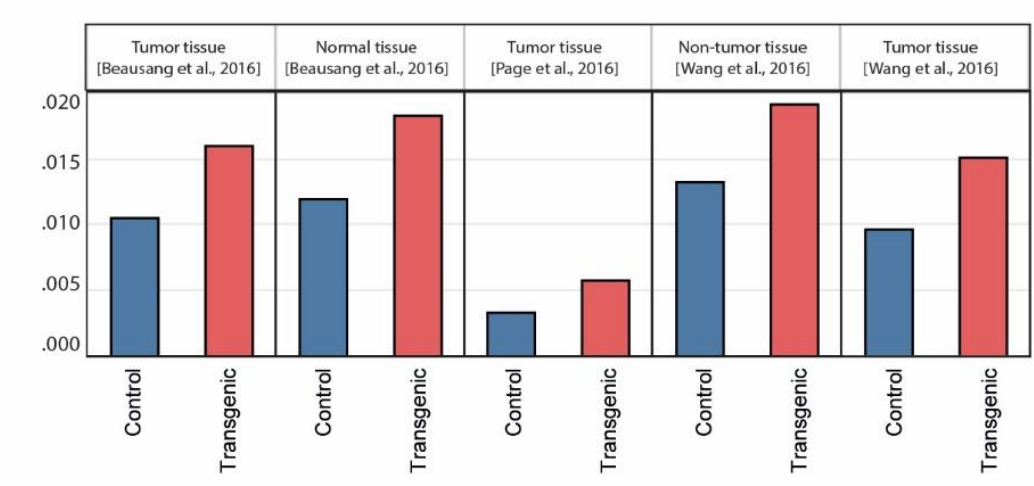

H
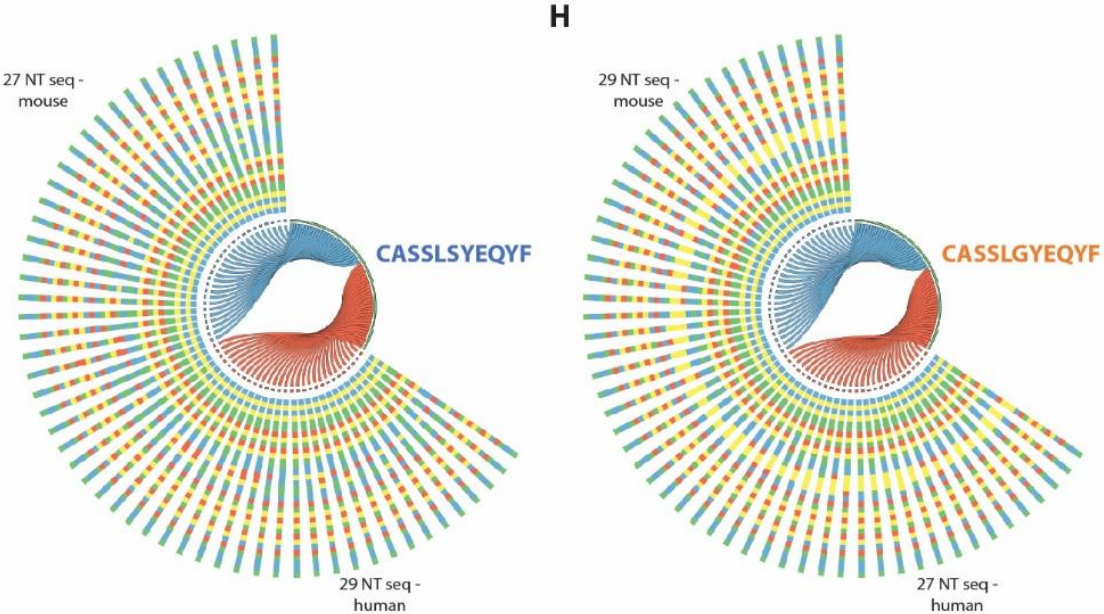
bioRxiv preprint doi: https://doi.org/10.1101/371260; this version posted August 6, 2018. The copyright holder for this preprint (which was not certified by peer review) is the author/funder. All rights reserved. No reuse allowed without permission.

Fig. 3. The connection between the mouse repertoire and human breast cancer. To learn more about the connection between the repertoires of mouse mammary tumor and human breast cancer, we studied the three published datasets described in (A). These include 50 breast cancer patients from different studies, in which different conditions and different tissues were studied. (B, C) The Public repertoires in the sub-populations of clones shared with human samples are shown using an NT view $(\boldsymbol{B})$ and an $A A$ view $(\boldsymbol{C}) .(\boldsymbol{D})$ The frequency of $C R$ clones that are shared with the human datasets is higher than the one measured in the mice alone. $(\boldsymbol{E})$ The percentile of clones that are shared between human and mouse samples shows that the Tg share more sequences with human samples (note that all human samples come from tumor-bearing individuals). (F) We ranked the 258 cross-species sequences according to their abundance in each sample. To visualize similarities between the ranking in each time point and the samples from early stage breast cancer patients as in Beausang et al., we stacked bars from each of the 258 ranking lines. The area of each bar has been determined so that it is reciprocal to (ranking in human X ranking in mouse). In that manner, if, in a specific time point, a clonotypes is ranked \#1 in mouse samples, and is also ranked \#1 in human samples, it would demonstrate the largest area. Color of sequences is preserved across bars, so that we see that three sequences dominate the similarity between samples: blue, orange and green. These sequences are included in Table 1, and, as the Table further indicates, have been previously associated with Melanoma, with Influenza, and with Diabetes. $(\boldsymbol{G}, \boldsymbol{H})$ The two highly ranked clones in the cross-species analyses are visualized for their CR sources. In the panel, each nucleotide sequence is connected to its translated amino-acid sequence. Edges are blue if they originate from a mouse sequence and red if they originate from a human sequence. As the panel shows, there is no overlap between sources for these two amino-acid sequences. (I) We used IGoR (Marcou, Mora, \& Walczak, 2018) (see Text) to learn of any differences between the populations of crossspecies sequences used in panels $(\boldsymbol{F}, \boldsymbol{G}, \boldsymbol{H})$ and the full collection of sequences. To do that, we plotted each sequence as a dot on the graph. Red dots represent cross-species sequences, blue dots represent the full set of all sequences, and green dots represent the $4700 \mathrm{NT}$ sequences that code for the 258 AA sequences that are shared across all time-points and with human samples. The vertical location of the dot is determined by its IGoR value and the horizontal location by its $C R$ value. The right-hand side curves present the histogram over the IGoR values, and the upper-side curves present the histograms over CR values. We used a Kolmogorov-Smirnov test to estimate p-value for the differences between distributions. Indeed, a highly significant $p$-value $\left(p<2.2 \times 10^{-16}\right)$ has been obtained, that demonstrates a large difference between the sequence populations. It is interesting to note, as shown by the three CR histograms on the upper side of the panel, that the CR values of these three populations of sequences also come from extremely different distributions $\left(p<2.2 \times 10^{-16}\right)$. We also highlighted the locations of the two sequences that are described in panels $(\boldsymbol{F}, \boldsymbol{G}, \boldsymbol{H})$. Although these two sequences differ by a single amino-acid. Their IGoR scores are very different.

that similarly ranked sequences occupy a larger area. For example, the largest area would be occupied by a sequence ranked 1 in both species. Figure $3 f$, at timepoint 5 , which is associated with early-tumors in mice, proves to be the time-point most similar to early-tumors in patients. We can also see that a small set of sequences (blue, orange, green, red in Figure 3f) dominates the chart. When we further study those specific sequences, we see that they are somewhat ubiquitous to TCR repertoire studies. They appear in other works, available from public datasets, and have been associated with Melanoma and Influenza (Table 1). Recent findings from other works show associations to these phenotypes (Laubli et al., 2018; Simoni et al., 2018).

Finally, to learn whether identical CDR3 AA sequences in mice and humans might originate from similar NT recombinations, we selected AA sequences that are highly ranked across species (blue and orange in Figure 3f), and compared their parent NT sequences. The results are shown in Figures $3 \mathrm{~g}$ and $3 \mathrm{~h}$, which connect each NT sequence to its end-result AA sequence. Edges are blue if they originate from a mouse sequence and red if they originate from a human sequence. We can see that the same amino-acid sequence originates from multiple NT sequences, and that the NT sequences have no overlap between the two species. The AA sequence in figure $3 \mathrm{~h}$ originates from 29 different DNA sequence in the mouse and from 27 different NT sequences in the human; and the AA sequence in Figure $3 \mathrm{~h}$ originates from 29 different NT sequences in mice and 27 different NT sequences in humans. Whatever the upstream NT recombination, the $\mathrm{CR}$ process strongly selects for a specific AA sequence. To learn more about the differences between the specifics groups of cross-species sequences and the general population of CDR3 sequences, we utilized IGoR, which is a recently developed tool (Marcou et al., 2018), that probabilistically annotates sequences. Figure 3 i shows the results of IGoR based analyses over the three groups discussed here: the collection of all sequences, the collection of all cross-species sequences, and the collection of the unique set of 258 cross-species sequences that are inherent to all samples, together with human samples. As the figure shows, the three curves are 1) significantly different from each other 2) Crossspecies sequences and the set of 258 sequences are associated with a distribution of relatively low probabilities on the one hand, and high CR (displayed at the top of the panel) distributions. These indicate that even though the sequences were not produced at higher probabilities, they were selected to appear as such. Of special interest, and highlighted in the figure, are the two sequences mentioned above, CASSLGYEQYF and CASSLSYEQYF, which, in spite of their single AA difference, appear with very different IGoR scores and with very high $C R$ values.

\begin{tabular}{lcccccc}
\hline CDR3 sequence & VDJdb & Peptide in VDJdb & $\begin{array}{c}\text { McPAS- } \\
\text { TCR }\end{array}$ & $\begin{array}{c}\text { Peptide in } \\
\text { McPAS-TCR }\end{array}$ & References & Ranking \\
\hline CASSYSYEQYF & - & - & Melanoma & - & (Britanova et al., 2014) & 1 \\
CASSPTGYEQYF & - & - & - & - & (Britanova et al., 2014; Seay et \\
al., 2016) & 2 \\
CASSLGYEQYF & - & ELAGIGILTV & Influenza & LPRRSGAAGA & $\begin{array}{c}\text { (Dandekar et al., 2016; Ko et } \\
\text { al., 2011; Page et al., 2016; } \\
\text { Schneider-Hohendorf et al., } \\
\text { 2016) }\end{array}$ \\
CASSLSYEQYF & - & & & & $\begin{array}{c}\text { 2016) } \\
\text { (Britanova et al., 2014; Rieux- } \\
\text { Laucat et al., 1998) }\end{array}$ \\
\hline
\end{tabular}

Table 1. CDR3 sequences that show cross-species, tumor-exclusive, behaviors 


\section{Discussion}

Investigation of the temporal behavior of TCR repertoires during mammary tumor development in mice, shows that they are dominated by Public clonotypes shared with human breast cancer patients. These shared, cross species TCR sequences are very likely to arise as a consequence of strong selection by antigen epitopes present in both mice and humans with breast tumors, as indicated by the continuous involvement of $\mathrm{CR}$. This $\mathrm{CR}$ and public clonotypes shape the unique structure of the repertoire in mice with tumors. The phenomenon of cross-species sequences has been demonstrated before (Madi et al., 2014) and is strongly associated here with Inclusive-public (public only in tumor) sequences. We also showed that early tumor development in mice shares a TCR repertoire with early tumors in human samples and that these specific sequences show high levels of $\mathrm{CR}$, that originate from different nucleotide sequences in mice and different nucleotide sequences in human, with no overlap, further indicating selection related to mammary tumors. Public databases (Shugay et al., 2018; Tickotsky, Sagiv, Prilusky, Shifrut, \& Friedman, 2017) show that these specific TCR sequences have previously been seen in connection with specific peptides reported in connection with other cancer types, and with CMV and influenza viruses. The sequences we report on here should be studied further in connection with responses to tumor immunotherapy. Modifying tumor repertoires to shift them in specific directions, which may be quantified according to some of the analytics we show here, may allow for novel treatments.

\section{Materials and Methods}

Mice. Transgenic Mice expressing the inactivated rat neu (Erbb2) oncogene under the transcriptional control of the mouse mammary tumor virus promoter were purchased from Jackson Laboratories (FVB/N-Tg(MMTVneu) 202 $\mathrm{Mul} / \mathrm{J})$. The female mice of this strain represent a mouse model of mammary tumor in humans, model of HER2/ Erbb2 / Neu human breast cancer (Guy et al., 1992). FVB/NJ strain with the same genetic background as the transgenic mice, serve as a non-transgenic control mouse that does not develop tumors. Mice were housed in accordance with all applicable laws and regulations following approval by the responsible animal care and ethical committee, under specific pathogen-free conditions. Mice were monitored by palpitation for tumor development monthly for up to 9 months.

Antibody staining and cell sorting. Blood was sampled from the retro-orbital sinus of 15 mice once per month for 8 time points (total of 120 samples). Mononuclear cells from the peripheral blood was isolated by density gradient centrifugation using Ficoll (Ficoll PaqueTM plus, GE Health Care), Single cell suspensions were prepared from thymus and spleen that were removed from each mouse at the end of the experiment. For cell sorting, cells were stained with the following fluorescently labeled monoclonal antibodies: anti-CD4 Pacific Blue (BD), anti-CD25 PE (eBioscience), antiCD44 APC (BD) and anti-CD62L PE-Cy7 (eBioscience) and viability using the Fixable Viability stain 450 (BD Horizon). Cell sorting was performed using FACS ARIA III sorter. CD4+ D44loCD62Lhi were sorted as naïve T cells. After sorting, cells were pelleted and resuspended with $300 \mu \mathrm{l}$ of RNAprotect cell reagent (Qiagen). Cells were stored at minus $80^{\circ} \mathrm{C}$ until RNA extraction. RNA was purified from RNAprotect-stabilized cells using the RNeasy Plus Mini Kit. After RNA extraction, samples were run on TapeStation to estimate quality.

High-throughput sequencing of the $\mathbf{T}$ cell repertoire. The method for high-throughput sequencing of the T cell repertoire was performed as previously described (Di Niro et al., 2015; Tsioris et al., 2015) . Briefly, RNA was reverse-transcribed into cDNA using a biotinylated oligo dT primer. An adaptor sequence was added to the 3 ' end of all cDNA, which contains the Illumina P7 universal priming site and a 17-nucleotide unique molecular identifier (UMI). Products were purified using streptavidin-coated magnetic beads followed by a primary PCR reaction using a pool of primers targeting the TCR $\alpha$ and TCR $\beta$ regions, as well as a sampleindexed Illumina $\mathrm{P} 7 \mathrm{C} 7$ primer. The TCR-specific primers contained tails corresponding to the Illumina P5 sequence. PCR products were then purified using AMPure XP beads. A secondary PCR was performed to add the Illumina $\mathrm{C} 5$ clustering sequence to the end of the molecule containing the constant region. The number of secondary PCR cycles was tailored to each sample to avoid entering plateau phase, as judged by a prior quantitative PCR analysis. Final products were purified, quantified with Agilent Tapestation and pooled in equimolar proportions, followed by high-throughput paired-end sequencing on the Illumina MiSeq platform. For sequencing, the Illumina 600 cycle kit was used with the modifications that 325 cycles was used for read 1, 6 cycles for the index reads, 300 cycles for read 2 and a $20 \%$ PhiX spike-in to increase sequence diversity.

Processing of raw sequencing data. Transforming raw reads to alpha and beta CDR3 sequences is done by using MiXCR, a universal framework that processes big immunome data from raw sequences and output quantitated clonotypes (Bolotin et al., 2015). MiXCR performs paired-end read merging and extracts human or animal BCR and TCR clonotypes providing corrections of erroneous sequences introduced by NGS (Kivioja et al., 2012; Vander Heiden et al., 2014; Yaari \& Kleinstein, 2015). 


\section{References}

Beausang, J. F., Wheeler, A. J., Chan, N. H., Hanft, V. R., Dirbas, F. M., Jeffrey, S. S., \& Quake, S. R. (2017). T cell receptor sequencing of early-stage breast cancer tumors identifies altered clonal structure of the $\mathrm{T}$ cell repertoire. Proc Natl Acad Sci U S A, 114(48), E10409-E10417. doi:10.1073/pnas.1713863114

Bolotin, D. A., Poslavsky, S., Mitrophanov, I., Shugay, M., Mamedov, I. Z., Putintseva, E. V., \& Chudakov, D. M. (2015). MiXCR: software for comprehensive adaptive immunity profiling. Nat Methods, 12(5), 380-381. doi:10.1038/nmeth.3364

Brentjens, R. J., Davila, M. L., Riviere, I., Park, J., Wang, X., Cowell, L. G., . . . Sadelain, M. (2013). CD19-targeted $T$ cells rapidly induce molecular remissions in adults with chemotherapy-refractory acute Iymphoblastic leukemia. Sci Transl Med, 5(177), 177 ra138. doi:10.1126/scitranslmed.3005930

Britanova, O. V., Putintseva, E. V., Shugay, M., Merzlyak, E. M., Turchaninova, M. A., Staroverov, D. B., . . . Chudakov, D. M. (2014). Age-related decrease in TCR repertoire diversity measured with deep and normalized sequence profiling. $J$ Immunol, 192(6), 2689-2698. doi:10.4049/jimmunol.1302064

Butterfield, L. H. (2015). Cancer vaccines. BMJ, 350, h988. doi:10.1136/bmj.h988

Dandekar, S., Wijesuriya, H., Geiger, T., Hamm, D., Mathern, G. W., \& Owens, G. C. (2016). Shared HLA Class I and II Alleles and Clonally Restricted Public and Private Brain-Infiltrating alphabeta $\mathrm{T}$ Cells in a Cohort of Rasmussen Encephalitis Surgery Patients. Front Immunol, 7, 608. doi:10.3389/fimmu.2016.00608

Di Niro, R., Lee, S. J., Vander Heiden, J. A., Elsner, R. A., Trivedi, N., Bannock, J. M., . . . Shlomchik, M. J. (2015). Salmonella Infection Drives Promiscuous B Cell Activation Followed by Extrafollicular Affinity Maturation. Immunity, 43(1), 120131. doi:10.1016/j.immuni.2015.06.013

Drake, C. G., Lipson, E. J., \& Brahmer, J. R. (2014). Breathing new life into immunotherapy: review of melanoma, lung and kidney cancer. Nat Rev Clin Oncol, 11(1), 24-37. doi:10.1038/nrclinonc.2013.208

Dunn, G. P., Bruce, A. T., Ikeda, H., Old, L. J., \& Schreiber, R. D. (2002). Cancer immunoediting: from immunosurveillance to tumor escape. Nat Immunol, 3(11), 991 998. doi:10.1038/ni1102-991

Fukui, Y., Hashimoto, O., Inayoshi, A., Gyotoku, T., Sano, T., Koga, T., . . . Sasazuki, T. (1998). Highly restricted $\mathrm{T}$ cell repertoire shaped by a single major histocompatibility complex-peptide ligand in the presence of a single rearranged $\mathrm{T}$ cell receptor beta chain. J Exp Med, 188(5), 897-907.

Gajewski, T. F., Schreiber, H., \& Fu, Y. X. (2013). Innate and adaptive immune cells in the tumor microenvironment. Nat Immunol, 14(10), 1014-1022. doi:10.1038/ni.2703

Galon, J., Costes, A., Sanchez-Cabo, F., Kirilovsky, A., Mlecnik, B., Lagorce-Pages, C., . . . Pages, F. (2006). Type, density, and location of immune cells within human colorectal tumors predict clinical outcome. Science, 313(5795), 1960-1964. doi:10.1126/science.1129139

Guy, C. T., Webster, M. A., Schaller, M., Parsons, T. J., Cardiff, R. D., \& Muller, W. J. (1992). Expression of the neu protooncogene in the mammary epithelium of transgenic mice induces metastatic disease. Proc Natl Acad Sci U S A, 89(22), 10578-10582.

Hanahan, D., \& Weinberg, R. A. (2011). Hallmarks of cancer: the next generation. Cell, 144(5), 646-674. doi:10.1016/j.cell.2011.02.013

Hoos, A., Ibrahim, R., Korman, A., Abdallah, K., Berman, D., Shahabi, V., . . . Humphrey, R. (2010). Development of ipilimumab: contribution to a new paradigm for cancer immunotherapy. Semin Oncol, 37(5), 533546. doi:10.1053/j.seminoncol.2010.09.015

Ino, Y., Yamazaki-Itoh, R., Shimada, K., Iwasaki, M., Kosuge, T., Kanai, Y., \& Hiraoka, N. (2013). Immune cell infiltration as an indicator of the immune microenvironment of pancreatic cancer. Br J Cancer, 108(4), 914923. doi:10.1038/bjc.2013.32 
Kalyan, S., \& Kabelitz, D. (2013). Defining the nature of human gammadelta T cells: a biographical sketch of the highly empathetic. Cell Mol Immunol, 10(1), 2129. doi:10.1038/cmi.2012.44

Kawakami, Y., Eliyahu, S., Delgado, C. H., Robbins, P. F., Sakaguchi, K., Appella, E., . . . Rosenberg, S. A. (1994). Identification of a human melanoma antigen recognized by tumor-infiltrating lymphocytes associated with in vivo tumor rejection. Proc Natl Acad Sci U S A, 91(14), 6458-6462.

Kivioja, T., Vaharautio, A., Karlsson, K., Bonke, M., Enge, M., Linnarsson, S., \& Taipale, J. (2012). Counting absolute numbers of molecules using unique molecular identifiers. Nat Methods, 9(1), 72-74. doi:10.1038/nmeth.1778

Ko, T. M., Chung, W. H., Wei, C. Y., Shih, H. Y., Chen, J. K., Lin, C. H., ... Hung, S. I. (2011). Shared and restricted T-cell receptor use is crucial for carbamazepine-induced StevensJohnson syndrome. J Allergy Clin Immunol, 128(6), 1266-1276 e1211. doi:10.1016/j.jaci.2011.08.013

Laubli, H., Balmelli, C., Kaufmann, L., Stanczak, M., Syedbasha, M., Vogt, D., . . Rothschild, S. I. (2018). Influenza vaccination of cancer patients during PD-1 blockade induces serological protection but may raise the risk for immune-related adverse events. $J$ Immunother Cancer, 6(1), 40. doi:10.1186/s40425-018-0353-7

Madi, A., Shifrut, E., Reich-Zeliger, S., Gal, H., Best, K., Ndifon, W., .. . Friedman, N. (2014). Tcell receptor repertoires share a restricted set of public and abundant CDR3 sequences that are associated with selfrelated immunity. Genome Res, 24(10), 1603-1612. doi:10.1101/gr.170753.113

Marcou, Q., Mora, T., \& Walczak, A. M. (2018). High-throughput immune repertoire analysis with IGoR. Nat Commun, 9(1), 561. doi:10.1038/s41467-018-02832-w

Pagana, K. D., \& Pagana, T. J. (2007). Mosby's diagnostic and laboratory test reference (8th ed.). St. Louis, Mo.: Mosby Elsevier.

Page, D. B., Yuan, J., Redmond, D., Wen, Y. H., Durack, J. C., Emerson, R., ... McArthur, H. L. (2016). Deep Sequencing of T-cell Receptor DNA as a Biomarker of Clonally
Expanded TILs in Breast Cancer after Immunotherapy. Cancer Immunol Res, $4(10), \quad 835-844$. doi:10.1158/2326 6066.CIR-16-0013

Pardoll, D. M. (2012). The blockade of immune checkpoints in cancer immunotherapy. Nat Rev Cancer, 12(4), 252-264. doi:10.1038/nrc3239

Pellicci, D. G., Uldrich, A. P., Le Nours, J., Ross, F., Chabrol, E., Eckle, S. B., . . Godfrey, D. I. (2014). The molecular bases of delta/alphabeta $T$ cell-mediated antigen recognition. J Exp Med, 211(13), 25992615. doi:10.1084/jem.20141764

Rieux-Laucat, F., Bahadoran, P., Brousse, N., Selz, F., Fischer, A., Le Deist, F., \& De Villartay, J. P. (1998). Highly restricted human T cell repertoire in peripheral blood and tissueinfiltrating lymphocytes in Omenn's syndrome. J Clin Invest, 102(2), 312-321. doi:10.1172/JCl332

Schneider-Hohendorf, T., Mohan, H., Bien, C. G., Breuer, J., Becker, A., Gorlich, D., . . Schwab, N. (2016). CD8(+) T-cell pathogenicity in Rasmussen encephalitis elucidated by large-scale T-cell receptor sequencing. Nat Commun, 7, 11153. doi:10.1038/ncomms11153

Seay, H. R., Yusko, E., Rothweiler, S. J., Zhang, L., Posgai, A. L., Campbell-Thompson, M., ... Brusko, T. M. (2016). Tissue distribution and clonal diversity of the $T$ and $B$ cell repertoire in type 1 diabetes. $\mathrm{JCl}$ Insight, 1(20), e88242. doi:10.1172/jci.insight.88242

Shugay, M., Bagaev, D. V., Zvyagin, I. V., Vroomans, R. M., Crawford, J. C., Dolton, G., . . . Chudakov, D. M. (2018). VDJdb: a curated database of T-cell receptor sequences with known antigen specificity. Nucleic Acids Res, 46(D1), D419-D427. doi:10.1093/nar/gkx760

Simoni, Y., Becht, E., Fehlings, M., Loh, C. Y., Koo, S. L., Teng, K. W. W., .. Newell, E. W. (2018). Bystander CD8(+) T cells are abundant and phenotypically distinct in human tumour infiltrates. Nature, 557(7706), 575-579. doi:10.1038/s41586-018-0130-2

Simpson, A. J., Caballero, O. L., Jungbluth, A., Chen, Y. T., \& Old, L. J. (2005). Cancer/testis antigens, gametogenesis and cancer. Nat 
Rev Cancer, 5(8), 615-625. doi:10.1038/nrc1669

Slingluff, C. L., Jr. (2011). The present and future of peptide vaccines for cancer: single or multiple, long or short, alone or in combination? Cancer J, 17(5), 343-350. doi:10.1097/PPO.0b013e318233e5b2

Sonpavde, G. (2017). PD-1 and PD-L1 Inhibitors as Salvage Therapy for Urothelial Carcinoma. $N$ Engl J Med, 376(11), 1073-1074. doi:10.1056/NEJMe1701182

Tickotsky, N., Sagiv, T., Prilusky, J., Shifrut, E., \& Friedman, N. (2017). McPAS-TCR: a manually curated catalogue of pathologyassociated $T$ cell receptor sequences. Bioinformatics, 33(18), 2924-2929. doi:10.1093/bioinformatics/bt×286

Tsioris, K., Gupta, N. T., Ogunniyi, A. O., Zimnisky, R. M., Qian, F., Yao, Y., ... Love, J. C. (2015). Neutralizing antibodies against West Nile virus identified directly from human B cells by single-cell analysis and next generation sequencing. Integr Biol (Camb), 7(12), 1587-1597. doi:10.1039/c5ib00169b

Vander Heiden, J. A., Yaari, G., Uduman, M., Stern, J. N., O'Connor, K. C., Hafler, D. A., . . . Kleinstein, S. H. (2014). pRESTO: a toolkit for processing high-throughput sequencing raw reads of lymphocyte receptor repertoires. Bioinformatics,
30(13),

1930-1932.

doi:10.1093/bioinformatics/btu138

Wang, T., Wang, C., Wu, J., He, C., Zhang, W., Liu, J., . . . Liu, X. (2017). The Different T-cell Receptor Repertoires in Breast Cancer Tumors, Draining Lymph Nodes, and Adjacent Tissues. Cancer Immunol Res, 5(2), 148-156. doi:10.1158/23266066.CIR-16-0107

Yaari, G., \& Kleinstein, S. H. (2015). Practical guidelines for B-cell receptor repertoire sequencing analysis. Genome Med, 7(1), 121. doi:10.1186/s13073-015-0243-2

Ye, B., Stary, C. M., Li, X., Gao, Q., Kang, C., \& Xiong, $X$. (2018). Engineering chimeric antigen receptor-T cells for cancer treatment. $\mathrm{Mol}$ Cancer, 17(1), 32. doi:10.1186/s12943018-0814-0

Yoshida, K., Cologne, J. B., Cordova, K., Misumi, M., Yamaoka, M., Kyoizumi, S., . . Kusunoki, Y. (2017). Aging-related changes in human Tcell repertoire over 20years delineated by deep sequencing of peripheral T-cell receptors. Exp Gerontol, 96, 29-37. doi:10.1016/j.exger.2017.05.015

Zhang, L., Conejo-Garcia, J. R., Katsaros, D., Gimotty, P. A., Massobrio, M., Regnani, G., ... Coukos, G. (2003). Intratumoral T cells, recurrence, and survival in epithelial ovarian cancer. N Engl J Med, 348(3), 203213. doi:10.1056/NEJMoa020177 